\title{
Comparison of indocyanine green fluorescence and methylene blue dye in the detection of sentinel lymph nodes in breast cancer
}

\author{
Zhenghui Wang ${ }^{1 \#}$, Yangyang Cui ${ }^{2 \#}$, Mingjie Zheng ${ }^{2}$, Han $\mathrm{Ge}^{2}$, Yue Huang ${ }^{2}$, Jinghui Peng ${ }^{2}, \mathrm{Hui}_{\mathrm{Xie}}^{2}$, \\ Shui Wang' \\ ${ }^{1}$ Department of Thyroid and Breast Surgery, The Second Hospital of Nanjing, Nanjing University of Chinese Medicine, Nanjing, China; \\ ${ }^{2}$ Department of Breast Surgery, The First Affiliated Hospital of Nanjing Medical University, Nanjing, China \\ Contributions: (I) Conception and design: Z Wang, H Xie, S Wang; (II) Administrative support: H Xie, S Wang; (III) Provision of study materials or \\ patients: Z Wang, M Zheng, H Ge, Y Huang, J Peng; (IV) Collection and assembly of data: Z Wang; (V) Data analysis and interpretation: Z Wang, \\ Y Cui; (VI) Manuscript writing: Z Wang; (VII) Final approval of manuscript: All authors. \\ \#These authors contributed equally to this work. \\ Correspondence to: Shui Wang, PhD; Hui Xie, PhD. Department of Breast Surgery, The First Affiliated Hospital of Nanjing Medical University, 300 \\ Guangzhou Road, Nanjing 210029, China. Email: ws0801@hotmail.com; Hxie@njmu.edu.cn.
}

Background: Previous studies have shown that sentinel lymph node biopsy (SLNB) can be successfully performed using methylene blue (MB); however, this method still has some drawbacks. Indocyanine green (ICG) fluorescence imaging, as a selective method, has the potential for guiding SLNB. This study aimed to compare the clinical sensitivity and efficacy between ICG and MB in SLNB in breast cancer.

Methods: A prospective study of 70 patients with biopsy-proven invasive breast cancer was conducted. Under the guidance of ICG and MB, administered by injection, SLNs were examined and removed. The detection rates, total number of SLNs detected, mean number of SLNs detected, and number of positive SLNs were compared between ICG and MB.

Results: The SLN detection rate was 100\% and 93\% (65/70) for ICG and MB, respectively. More SLNs were detected in the ICG group (243) than in the MB group (169). The mean number of SLNs detected with ICG and MB was 3.5 \pm 1.73 and 2.4 \pm 1.49 , respectively. Moreover, there was a statistically significant difference between the number of SLNs detected using the two methods $(\mathrm{t}=6.648, \mathrm{P}<0.05)$. Additionally, SLN metastasis was detected in 18 patients using ICG and 14 patients using MB; these patients immediately underwent axillary lymph node dissection (ALND). No postoperative complications were reported.

Conclusions: ICG demonstrated a higher detection rate and better accuracy, as well as a lower false negative rate, than MB in detecting SLNs in breast cancer. ICG has potential as an alternative tool that could be clinically applied to detect SLNs in breast cancer patients.

Keywords: Breast cancer; sentinel lymph node (SLN); indocyanine green (ICG); methylene blue (MB); fluorescence imaging

Submitted Jul 19, 2020. Accepted for publication Sep 27, 2020.

doi: 10.21037/gs-20-671

View this article at: http://dx.doi.org/10.21037/gs-20-671

\section{Introduction}

The incidence of breast cancer, one of the most common malignant tumors in women, has risen continuously in recent years (1). Surgery is the main mode of treatment for breast cancer at present. In the past, the majority of breast cancer patients required axillary lymph node dissection (ALND). However, ALND can lead to various complications of the upper extremity that significantly impact patients' quality of life, such as edema, pain, numbness, and movement disorders. Sentinel lymph node biopsy (SLNB) has gradually become routine in breast cancer management for staging axillary lymph node 
status. Patients who are found to have negative SLNs after undergoing SLNB may not need to undergo ALND, which significantly reduces the risk of complications and speeds up the patient's return to normal life $(2,3)$. SLNB in breast cancer is usually performed using methylene blue (MB), radioisotope, or a combination of the two $(4,5)$. High rates of sentinel lymph node detection have been reported with all of these methods; however, the high costs, necessity for training, and radiation exposure associated with radioisotope limit its use. In contrast, blue dye does not possess limitations and has become widely used in SLNB. Blue dye can be injected easily and allows SLNs to be clearly visualized. However, studies have reported a reduced detection rate when $\mathrm{MB}$ is used alone, along with a higher false negative rate, which has given rise to concerns regarding potential disease recurrence and long-term prognosis $(6,7)$.

Evidence exists to suggest that SLN detection guided by indocyanine green (ICG) fluorescence may serve as a highly sensitive modality for SLNB. This technique combines the advantages of both radioisotope and MB to enable transcutaneous real-time lymphography and SLN staining (8-15). Therefore, this study aimed to evaluate the potential value of ICG as a novel tracer for guiding SLNB by comparing its clinical performance with that of $\mathrm{MB}$ in SLNB in patients with breast cancer.

We present the following article in accordance with the STROBE reporting checklist (available at http://dx.doi. org/10.21037/gs-20-671).

\section{Methods}

\section{Patients}

Between May 2018 and October 2018, 70 breast cancer patients were diagnosed at the First Affiliated Hospital of Nanjing Medical University. The diagnoses of all patients were confirmed by core needle biopsy or open biopsy. All procedures performed in this study involving human participants were in accordance with the Declaration of Helsinki (as revised in 2013). The study was approved by the medical ethics committee of the First Affiliated Hospital of Nanjing Medical University (No. 2018MD-077). Patients meeting any of the following criteria were excluded: previous radiotherapy for breast cancer; unable to tolerate surgery; previous axillary surgery; inflammatory breast cancer; distant lymph node metastasis; hypersensitivity to ICG or iodine, or accompanied by other diseases. Written informed consent was obtained from all patients before participation in the study.

\section{ICG administration and quantitative equipment}

ICG was purchased from Yichuang Pharmaceutical Co., Ltd. (Dandong, China). Fluorescence intensity and images were captured using a near-infrared fluorescence (NIF) image-guided system (REAL-IGS, Nanjing Nuoyuan Medical Devices Co., Ltd., Nanjing, China) integrated with a NIR spectrometer (Maya2000 Pro, Ocean Optics, Dunedin, Florida). MB was bought from Jumpcan Pharmaceutical Co., Ltd. (Hubei, China).

\section{Quantification of injection dose and the operation procedure}

Two different sites in the upper outer part of the areola were selected, and $1 \mathrm{~mL} \mathrm{MB}$ and $1 \mathrm{~mL}$ ICG were subcutaneously injected, respectively. There is no mutual interference between ICG and MB. The breast was massaged for 10 20 seconds to ensure the tracer entered quickly into the breast stroma and lymphatic vessels. Then, the operation room light was turned off. The device was turned on, and the lymphatic vessels from the ICG injection site to the site where the axillary fluorescence had developed were observed. An incision was made 1-2 $\mathrm{cm}$ from the point where the fluorescence in the lymphatic vessels disappeared, in the direction of the axilla.

Observe and count:

- Fluorescence: SpectraSuite software (Omni Driver, Ocean Optics, Dunedin, Florida) was used to measure fluorescence intensity. Background interference was eliminated before the measurements were taken. The highlighted lymph nodes were counted. Spectral measurement and analysis were performed at the marked incision using a handheld laser probe.

* MB: blue-stained lymph nodes in the tissue at the marked incision were observed simultaneously. All lymph nodes stained blue were marked and counted.

All detected lymph nodes were removed and submitted to the Department of Pathology for pathological diagnosis. Mammectomies and other surgical procedures were carried out where necessary, in accordance with clinical standards. 
Table 1 Patient clinical characteristics

\begin{tabular}{|c|c|}
\hline Characteristic & N (\%) \\
\hline \multicolumn{2}{|l|}{ Age } \\
\hline$\leq 55$ & $53(75.7)$ \\
\hline$>55$ & $17(24.3)$ \\
\hline \multicolumn{2}{|l|}{ Tumor location } \\
\hline Right & $39(55.7)$ \\
\hline Left & $31(44.3)$ \\
\hline \multicolumn{2}{|l|}{ TNM staging } \\
\hline $\mathrm{T} 1$ & $31(44.3)$ \\
\hline $\mathrm{T} 2$ & $36(51.4)$ \\
\hline T3 & $3(4.3)$ \\
\hline \multicolumn{2}{|l|}{ Tumor grade } \\
\hline I & $8(11.4)$ \\
\hline II & $46(65.7)$ \\
\hline III & $16(22.9)$ \\
\hline \multicolumn{2}{|c|}{ Molecular subtype } \\
\hline LuminalA & $21(30.0)$ \\
\hline LuminalB & $31(44.3)$ \\
\hline Her-2 positive & $10(14.3)$ \\
\hline Triple negative & $8(11.4)$ \\
\hline \multicolumn{2}{|l|}{ Estrogen receptor } \\
\hline Positive & $53(75.7)$ \\
\hline Negative & $17(24.3)$ \\
\hline \multicolumn{2}{|c|}{ Progesterone receptor } \\
\hline Positive & $47(67.1)$ \\
\hline Negative & $23(32.9)$ \\
\hline \multicolumn{2}{|l|}{ Her-2/neu } \\
\hline Positive & $19(27.1)$ \\
\hline Negative & $51(72.9)$ \\
\hline \multicolumn{2}{|l|}{ Ki-67 } \\
\hline$\leq 15 \%$ & $26(37.1)$ \\
\hline$>15 \%$ & 44 (62.9) \\
\hline
\end{tabular}

\section{Statistical analyses}

All statistical analyses were performed using the SPSS ${ }^{\circledR}$ statistical package, version 22.0 (SPSS Inc., Chicago, IL, USA) for Windows ${ }^{\circledR}$. Quantitative variables were presented as the mean \pm standard deviation (SD), while qualitative variables were presented as the numbers and percentages of cases. Paired-sample $t$-tests were performed to compare the number of SLNs detected by ICG and MB in each patient. $\mathrm{P}<0.05$ was considered statistically significant.

\section{Results}

Between May 2018 and October 2018, 70 women with breast cancer were enrolled in this study before undergoing surgery. For all eligible patients, SLNB was performed first, and ALND was performed based on the results of SLNs examination during surgery. All of the subjects underwent SLNB using both ICG and MB. The patients had a median age of $49.1 \pm 9.7$ years (range, 27-75 years). Table 1 summarizes the patients' clinical characteristics.

The application of ICG NIF image-guided surgery in breast cancer is shown in Figure 1.

The overall SLN detection rate was $100 \%$ (70/70) for ICG and $93 \%$ (65/70) for MB. The total number of SLNs detected by ICG and MB was 243 and 169, respectively. The mean number of SLNs removed was $3.5 \pm 1.73$ for ICG, compared with $2.4 \pm 1.49$ for MB. The number of SLNs removed with ICG fluorescence was significantly higher than that with MB. There was a statistically significant difference in the number of SLNs detected using the two different methods $(\mathrm{t}=6.648, \mathrm{P}<0.05)$. The results of pathology showed that the positive SLN detection rate with ICG was $25.7 \%(18 / 70)$, which was significantly higher than the $21.5 \%(14 / 65)$ with MB. As a result, 18 patients immediately underwent ALND, and lymph node micrometastases were found in the tissue samples postoperatively. No metastases were found by intraoperative or postoperative pathology in the samples of the remaining 52 patients. Therefore, the addition of ICG was associated with a significant increase in the detection rate of positive SLNs compared with $\mathrm{MB}$ alone. All data relating to the detection of SLNs are summarized in Tables 2,3.

No acute allergic reactions to ICG injections were reported, and no adverse events, such as nausea, vomiting, and pain, were recorded.

\section{Discussion}

$\mathrm{MB}$ and/or radioisotope are the main techniques used to detect SLNs in breast cancer. Dual localization techniques that employ both $\mathrm{MB}$ and radioisotope are effective in 

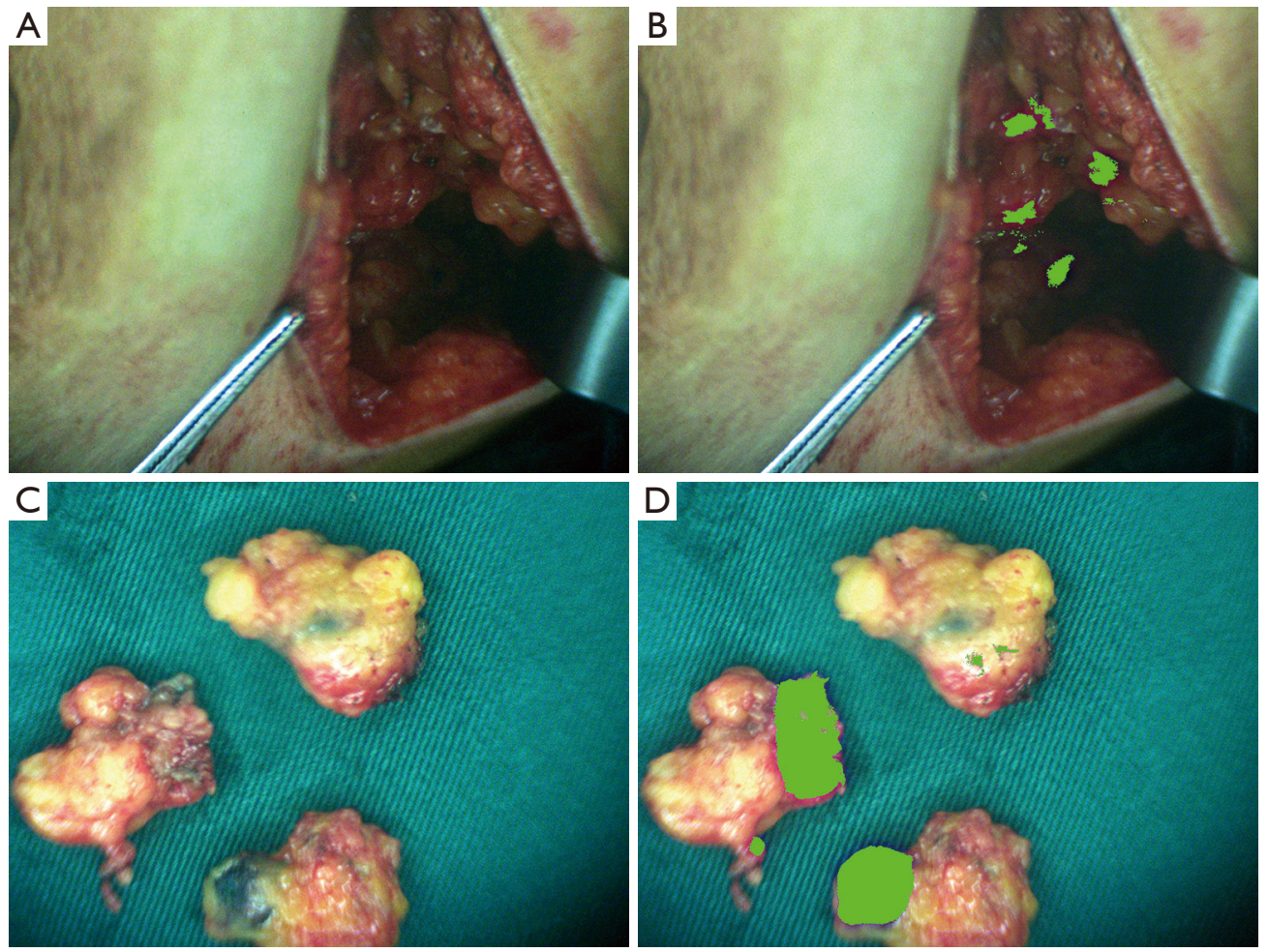

Figure 1 Sentinel lymph node detection by ICG fluorescence imaging system during surgery. (A) A skin incision at the marked area. (B) Fluorescence image of axillary tissue under the fluorescence imaging device, with green indicating the sentinel lymph node. (C) Sentinel lymph node specimens. (D) Fluorescence image of sentinel lymph node using the fluorescence imaging device. ICG, indocyanine green.

Table 2 The detection of SLNs in 70 patients

\begin{tabular}{lcc}
\hline Item & ICG & MB \\
\hline SLN detection rate & $100 \%(70 / 70)$ & $93 \%(65 / 70)$ \\
Total no. of SLNs & 243 & 169 \\
Mean no. of SLNs & $3.5 \pm 1.73$ & $2.4 \pm 1.49$ \\
SLN-positive patients & 18 & 14 \\
SLN-positive detection rate & $25.7 \%(18 / 70)$ & $21.5 \%(14 / 65)$ \\
\hline
\end{tabular}

SLN, sentinel lymph node; ICG, indocyanine green; MB, methylene blue.

detecting SLNs. MB is widely used in China, owing to its convenience and low cost; however, due to radiation exposure as well as high equipment costs, radioisotope techniques are rarely adopted in hospitals. The present study aimed to assess whether the addition of ICG to MB could improve the SLN detection rate in patients with breast cancer $(16,17)$. In addition to enhancing detection performance, ICG may improve accuracy. First and foremost, the high sensitivity of ICG was found to contribute to the harvesting of additional SLNs that undetected by MB (18-20). In this study, the numbers of SLNs detected by ICG apparently exceeded those of SLNs detected by $\mathrm{MB}(\mathrm{t}=6.648, \mathrm{P}=0.000<0.05)$. The higher sensitivity of ICG may be attributable to its higher visibility when observed by high-resolution near-infrared equipment. Moreover, this higher degree of visibility may also have contributed to the improved detection rate of positive nodes ( $\mathrm{ICG}=25.7 \%>\mathrm{MB}=21.5 \%$ ), which may contribute to reducing false-negative assessments in axillary nodes and generating beneficial treatment strategies. For instance, some patients with no SLNs detected by blue staining do not require ALND; however, the identification of additional positive SLNs by ICG enables them to avoid being missed by ALND, which reduces the probability of recurrence and metastasis, and improves patient prognosis. Meanwhile, a number of studies have suggested that excessive ALND increases the risk of postoperative complications of the upper limb, while its effects on prognosis are unknown (21-25). Therefore, additional clinical trials should be 
Table 3 Results of paired $t$-tests to compare the number of SLNs detected by ICG and MB in each patient

\begin{tabular}{lccccc}
\hline Sample size & Mean value & SD & SEM & $t$ & P \\
\hline 70 & 1.043 & 1.313 & 0.157 & 6.648 & 0.000 \\
& & & & $<.050$ \\
\hline
\end{tabular}

SLN, sentinel lymph node; ICG, indocyanine green; MB, methylene blue; SD, standard deviation; SEM, standard error of the mean.

conducted to establish any potential benefits for patients (26). The high identification rate and accurate diagnostic performance of ICG fluorescence potentially provide an alternative method for tracing SLNs, and ICG may eventually replace the traditional applications of blue staining and radioactive agents as the preferred method. Our findings are consistent with those of previous studies (27-30).

As previously reported, ICG-guided SLNB has several limitations. Firstly, the tissue penetration capacity of NIR fluorescence is inferior to that of gamma rays, which may lead to worse overall performance in patients, especially in those who are overweight $(31,32)$. Furthermore, macrometastasis can limit the diffusion of ICG from the lymphatic channels to the lymph nodes. In such cases, ICG cannot penetrate into the initial lymph node or escape to the second echelon or false sentinel lymph node (33-35). Such disadvantages weaken the detection capability of ICG.

The current study had several limitations. Firstly, the sample size was small, the exclusion criteria did not eliminate individual variation, and the statistical efficiency needed to be further optimized. Future studies should address these issues to decrease confounding bias. Secondly, after all fluorescent nodes have been detected during the procedure, surgeons might tend to end the procedure, rather than continuing to search for more blue nodes. This might result in an overestimation of the accuracy of ICG and underestimation of the efficacy of blue staining.

Adverse effects, such as the temporary skin staining, permanent tattooing, and subcutaneous nodules at the injection sites sometimes result in certain, but acceptable, degrees of anxiety in some patients.

This study presented the high detection rate and superiority of ICG when used to detect SLNs in patients with breast cancer. ICG fluorescence should be used in hospitals and can be used as a substitute for $\mathrm{MB}$ or radioisotope in the absence of radioactive agents.

\section{Conclusions}

ICG has a high SLN detection rate and improves the mapping performance. As a tracing modality, ICG demonstrates great potential as a novel alternative to the traditional standard mapping methods.

\section{Acknowledgments}

Funding: None.

\section{Footnote}

Reporting Checklist: The authors have completed the STROBE reporting checklist. Available at http://dx.doi. org/10.21037/gs-20-671

Data Sharing Statement: Available at http://dx.doi. org/10.21037/gs-20-671

Conflicts of Interest: All authors have completed the ICMJE uniform disclosure form (available at http://dx.doi. org/10.21037/gs-20-671). The authors have no conflicts of interest to declare.

Ethical Statement: The authors are accountable for all aspects of the work in ensuring that questions related to the accuracy or integrity of any part of the work are appropriately investigated and resolved. All procedures performed in this study involving human participants were in accordance with the Declaration of Helsinki (as revised in 2013). The study was approved by the medical ethics committee of the First Affiliated Hospital of Nanjing Medical University (No. 2018-MD-077). Written informed consent was obtained from all patients before participation in the study.

Open Access Statement: This is an Open Access article distributed in accordance with the Creative Commons Attribution-NonCommercial-NoDerivs 4.0 International License (CC BY-NC-ND 4.0), which permits the noncommercial replication and distribution of the article with the strict proviso that no changes or edits are made and the 
original work is properly cited (including links to both the formal publication through the relevant DOI and the license). See: https://creativecommons.org/licenses/by-nc-nd/4.0/.

\section{References}

1. Bray F, Ferlay J, Soerjomataram I, et al. Global cancer statistics 2018: GLOBOCAN estimates of incidence and mortality worldwide for 36 cancers in 185 countries. CA Cancer J Clin 2018;68:394-424.

2. He Z, Zhou Y, Wang F, et al. Clinical value of postoperative sentinel lymph node biopsy. Ann Transl Med 2019;7:683.

3. Chirappapha P, Arunnart M, Lertsithichai P, et al. Evaluation the effect of preserving intercostobrachial nerve in axillary dissection for breast cancer patient. Gland Surg 2019;8:599-608.

4. Ahmed M, Purushotham AD, Douek M, et al. Novel techniques for sentinel lymph node biopsy in breast cancer: a systematic review. Lancet Oncol 2014;15:e351-62.

5. He PS, Li F, Li GH, et al. The combination of blue dye and radioisotope versus radioisotope alone during sentinel lymph node biopsy for breast cancer: a systemic review. BMC Cancer 2016;16:107.

6. Li H, Jun Z, Zhi-Cheng G, et al. Factors that affect the false negative rate of sentinel lymph node mapping with methylene blue dye alone in breast cancer. J Int Med Res 2019;47:4841-53.

7. Li J, Chen X, Qi M, et al. Sentinel lymph node biopsy mapped with methylene blue dye alone in patients with breast cancer: A systematic review and meta-analysis. PLoS One 2018;13:e204364.

8. Hokimoto N, Sugimoto T, Namikawa T, et al. A novel color fluorescence navigation system for intraoperative transcutaneous lymphatic mapping and resection of sentinel lymph nodes in breast cancer: comparison with the combination of gamma probe scanning and visible dye methods. Oncology 2018;94:99-106.

9. Takada M, Takeuchi M, Suzuki E, et al. Real-time navigation system for sentinel lymph node biopsy in breast cancer patients using projection mapping with indocyanine green fluorescence. Breast Cancer 2018;25:650-5.

10. Sorrentino L, Sartani A, Pietropaolo G, et al. A novel indocyanine green fluorescence-guided video-assisted technique for sentinel node biopsy in breast cancer. World J Surg 2018;42:2815-24.

11. Hidar S. Use of indocyanine green for detecting sentinel lymph nodes in breast cancer: letter to the editor. World J
Surg Oncol 2017;15:70.

12. Sugie T, Ikeda T, Kawaguchi A, et al. Sentinel lymph node biopsy using indocyanine green fluorescence in earlystage breast cancer: a meta-analysis. Int J Clin Oncol 2017;22:11-7.

13. Valente SA, Al-Hilli Z, Radford DM, et al. Near infrared fluorescent lymph node mapping with indocyanine green in breast cancer patients: a prospective trial. J Am Coll Surg 2019;228:672-8.

14. Sugie T. Current status and future perspectives of the navigation surgery in breast cancer - the clinical utility of sentinel lymph node biopsy using ICG. Gan To Kagaku Ryoho 2018;45:1139-43.

15. Lin J, Lin LS, Chen DR, et al. Indocyanine green fluorescence method for sentinel lymph node biopsy in breast cancer. Asian J Surg 2020. [Epub ahead of print].

16. Yuan L, Qi X, Zhang Y, et al. Comparison of sentinel lymph node detection performances using blue dye in conjunction with indocyanine green or radioisotope in breast cancer patients: a prospective single-center randomized study. Cancer Biol Med 2018;15:452-60.

17. Qin X, Yang M, Zheng X. Comparative study of indocyanine green combined with blue dye with methylene blue only and carbon nanoparticles only for sentinel lymph node biopsy in breast cancer. Ann Surg Treat Res 2019;97:1-6.

18. Shen S, Xu Q, Zhou Y, et al. Comparison of sentinel lymph node biopsy guided by blue dye with or without indocyanine green in early breast cancer. J Surg Oncol 2018;117:1841-7.

19. Ji Y, Luo N, Jiang Y, et al. Clinical utility of the additional use of blue dye for indocyanine green for sentinel node biopsy in breast cancer. J Surg Res 2017;215:88-92.

20. Ban EJ, Lee JS, Koo JS, et al. How many sentinel lymph nodes are enough for accurate axillary staging in t1-2 breast cancer? J Breast Cancer 2011;14:296-300.

21. Ebner F, Wöckel A, Schwentner L, et al. Does the number of removed axillary lymphnodes in high risk breast cancer patients influence the survival? BMC Cancer 2019;19:90.

22. Yuan Q, Wu G, Xiao SY, et al. Identification and Preservation of Arm Lymphatic System in Axillary Dissection for Breast Cancer to Reduce Arm Lymphedema Events: A Randomized Clinical Trial. Ann Surg Oncol 2019;26:3446-54.

23. Galimberti V, Cole BF, Viale G, et al. Axillary dissection versus no axillary dissection in patients with breast cancer and sentinel-node micrometastases (IBCSG 23-01):10year follow-up of a randomized, controlled phase 3 trial. 
Lancet Oncol 2018;19:1385-93.

24. Nadeem RM. The feasibility of a randomized controlled trial for the axillary management of a select group of invasive breast cancer patients: SLNB vs no-SLNB. Breast Cancer 2015;22:343-9.

25. Abbaci M, Conversano A, De Leeuw F, et al. Near-infrared fluorescence imaging for the prevention and management of breast cancer-related lymphedema: a systematic review. Eur J Surg Oncol 2019;45:1778-86.

26. Vaz T, Costa S, Peleteiro B. Fluorescence-guided sentinel lymph node biopsy in breast cancer: detection rate and diagnostic accuracy. Acta Med Port 2018;31:706-13.

27. Pitsinis V, Wishart GC. Comprison of indocyanine green fluorescence and blue dye methods in dection of sentinel lymph nodes in early-stage breast cancer. Ann Surg Oncol 2017;24:581-2.

28. Guo J, Yang H, Wang S, et al. Comparison of sentinel lymph node biopsy guided by indocyanine green, blue dye, and their combination in breast cancer patients: a prospective cohort study. World J Surg Oncol 2017;15:196.

29. He K, Chi C, Kou D, et al. Comparison between the indocyanine green fluorescence and blue dye methods for sentinel lymph node biopsy using novel fluorescence image-guided resection equipment in different types of hospitals. Transl Res 2016;178:74-80.

Cite this article as: Wang Z, Cui Y, Zheng M, Ge H, Huang Y, Peng J, Xie H, Wang S. Comparison of indocyanine green fluorescence and methylene blue dye in the detection of sentinel lymph nodes in breast cancer. Gland Surg 2020;9(5):14951501. doi: $10.21037 /$ gs-20-671
30. Liu J, Huang L, Wang N, et al. Indocyanine green detects sentinel lymph nodes in early breast cancer. J Int Med Res 2017;45:514-24.

31. Goonawardena J, Yong C, Law M. Use of indocyanine green fluorescence compared to radioisotope for sentinel lymph node biopsy in early-stage breast cancer: systematic review and meta-analysis. Am J Surg 2020;220:665-76.

32. Guenane Y, Gorj M, Nguyen V, et al. Evaluation of green indocyanine interest compared to Technetium in sentinel lymph node detection in breast cancer. Ann Chir Plast Esthet 2016;61:806-10.

33. Mazouni C, Koual M, De Leeuw F, et al. Prospective evaluation of the limitations of near-infrared imaging in detecting axillary sentinel lymph nodes in primary cancer. Breast J 2018;24:1006-9.

34. Ma X, Wen S, Liu B, et al. Relationship between Upper Extremity Lymphatic Drainage and Sentinel Lymph Nodes in Patients with Breast Cancer. J Oncol 2019;2019:8637895.

35. Giuliano AE, Ballman KV, Mccall L, et al. Effect of axillary dissection among women with invasive breast cancer and sentinel node metastasis: The ACOSOG Z011(Alliance) Randomized Clinical Trial. JAMA 2017; 318:918-26.

(English Language Editor: J. Reynolds) 